\title{
A STUDY OF FUNCTION SPACES BY SPECTRAL SEQUENCES $\left({ }^{1}\right)$
}

\author{
BY \\ HERBERT FEDERER
}

1. Introduction. Given two topological spaces $X$ and $Y$ and a continuous map $v: X \rightarrow Y$, it is natural to ask how the homotopy groups of the function space $Y^{X}$ with the base point $v$ are algebraically related to homotopy type invariants of $X, Y$ and $v$. Assuming that $X$ is a finite dimensional cellular space and that $Y$ is simple in all dimensions, we shall partially answer this question by constructing an exact couple with an associated spectral sequence whose second term is the direct sum of the cohomology groups of $X$ with coefficients in the homotopy groups of $Y$, and whose limit group is the direct sum of the composition factors of certain normal chains for the homotopy groups of $Y^{x}$. Of course in order to compute this limit group in specific cases one must know enough about the homotopy type of $X, Y$ and $v$ to interpret the higher differential operators in terms of more familiar operations, as we shall do in some illustrative examples. We also construct examples to show that $\pi_{1}\left(Y^{x}, v\right)$ may be non-abelian despite the simplicity of $Y$, a fact which forces us to allow non-abelian groups in certain parts of our exact couples $\left({ }^{2}\right)$.

2. Basic facts about exact couples. We slightly generalize the definition first given by Massey in [1] and say that an exact couple

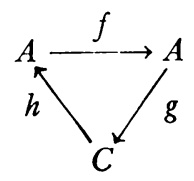

is an algebraic system consisting of a group $A$ (not necessarily abelian), an abelian group $C$, and three homomorphisms $f, g, h$ as in the diagram with image $f=$ kernel $g$, image $g=$ kernel $h$, image $h=$ kernel $f$.

It follows that $g \circ h$ is an endomorphism of $C$ with $(g \circ h)^{2}=0$, whence one may form the homology group

$$
H(C, g \circ h)=\text { kernel }(g \circ h) / \text { image }(g \circ h) \text {. }
$$

Presented to the Society, November 12, 1955; received by the editors December 27, 1955.

(1) This work was supported in part by a research grant from the National Science Foundation.

(2) The referee has called our attention to the related work of M. G. Barratt, Track groups I, Proc. London Math. Soc. (3) vol. 5 (1955) pp. 71-106, which contains many interesting results on homotopy groups of function spaces. 
Then the derived exact couple

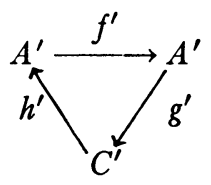

is defined by letting

$$
A^{\prime}=\text { image } f, \quad C^{\prime}=H(C, g \circ h), \quad f^{\prime}=f \mid A^{\prime}
$$

and by requiring commutativity of the diagram:

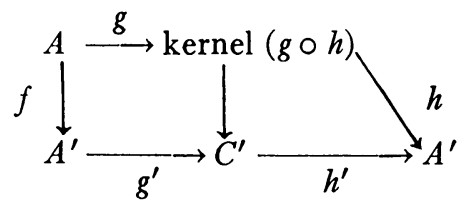

Applying the process of derivation $n$ times one obtains the $n$th derived couple

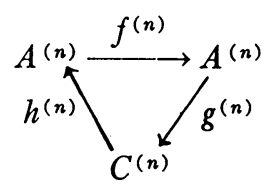

which may also be characterized directly in terms of the original exact couple as follows: Let $\left(^{3}\right)$

$$
\begin{aligned}
A^{(n)} & =\text { image } f^{n}, & f^{(n)} & =f \mid A^{(n)}, \\
Z^{(n)} & =h^{-1}\left(A^{(n)}\right), & B^{(n)} & =g\left(\text { kernel } f^{n}\right) !
\end{aligned}
$$

Then $B^{(n)} \subset Z^{(n)}$ and there exists a homomorphism $\phi^{(n)}$ such that the sequence

$$
\{0\} \longrightarrow B^{(n)} \longrightarrow Z^{(n)} \stackrel{\phi^{(n)}}{\longrightarrow} C^{(n)} \longrightarrow\{0\}
$$

is exact and such that the diagram

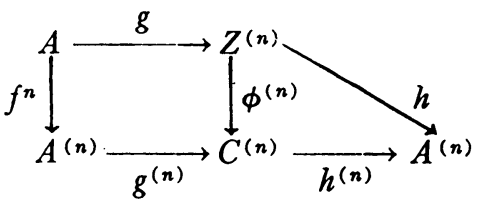

is commutative.

(3) Here $f^{n}$ is the $n$-iterate of the function $f$, while $f^{(n)}$ is one of the homomorphisms in the $n$th derived couple. 
The groups

$$
C^{(n)} \simeq Z^{(n)} / B^{(n)}
$$

with the differential operators $d^{(n)}=g^{(n)} \circ h^{(n)}$ form a spectral sequence with the limit group

$$
C^{(\infty)} \simeq \bigcap_{n=0}^{\infty} Z^{(n)} / \bigcup_{n=0}^{\infty} B^{(n)}
$$

3. Conventions concerning homotopy groups. Let $I^{\infty}$ be the set of all infinite sequences $\xi=\left(\xi_{1}, \xi_{2}, \xi_{3}, \cdots\right)$ such that $0 \leqq \xi_{i} \leqq 1$ for $i=1,2,3, \cdots$ and $\xi_{i}=0$ for all but finitely many $i$. For $n=0,1,2, \cdots$ let

$$
\begin{aligned}
& I^{n}=I^{\infty} \cap\left\{\xi \mid \xi_{i}=0 \text { for } i>n\right\}, \\
& \dot{I}^{n}=I^{n} \cap\left\{\xi \mid \xi_{i}=0 \text { or } \xi_{i}=1 \text { for some } i \leqq n\right\}, \\
& J^{n}=\dot{I}^{n+1} \cap\left\{\xi \mid \xi_{i}=0 \text { or } \xi_{i}=1 \text { for some } i \leqq n, \text { or } \xi_{n+1}=1\right\} .
\end{aligned}
$$

Thus $I^{0}=\{\theta\}$, where $\theta$ is the point all of whose coordinates are 0 .

For any space $S$ and any point $s \in S$ we regard $\pi_{n}(S, s)$ in the usual manner (see [6]) as the set of all arcwise components of the function space

$$
(S, s)^{\left(I^{n}, i^{n}\right)} \text {. }
$$

Here we include the case in which $n=0$; while in general $\pi_{0}(S, s)$ is not naturally endowed with a group structure, such a structure will conveniently appear in our particular applications. We recall that the maps

$$
(S, s)^{\left(I^{n}, i^{n}\right)} \leftarrow(S, s)^{\left(\dot{i}^{n+1}, J^{n}\right)} \rightarrow(S, s)^{\left(\dot{i}^{n+1}, \theta\right)}
$$

corresponding to the inclusions $\left(I^{n}, \dot{I}^{n}\right) \subset\left(\dot{I}^{n+1}, J^{n}\right) \supset\left(\dot{I}^{n+1}, \theta\right)$ induce one-toone correspondences between the arcwise components of these three function spaces. Thus any map $u$ of $\left(I^{n}, \dot{I}^{n}\right)$ or $\left(\dot{I}^{n+1}, \theta\right)$ into $(S, s)$ represents unambiguously an element $\langle u\rangle$ of $\pi_{n}(S, s)$.

We shall use the following form of the homotopy addition theorem (where $n \geqq 1)$ : For $i=1,2, \cdots$, m let $\alpha_{i}: I^{n} \rightarrow \dot{I}^{n+1}$ be a continuous map which is univalent on $I^{n}-\dot{I}^{n}$ and let $R_{i}=\alpha_{i}^{\prime}\left(I^{n}-\dot{I}^{n}\right)$. Suppose the sets $R_{1}, R_{2}, \cdots, R_{m}$ are disjoint and $\beta: \dot{I}^{n+1} \rightarrow S$ is a continuous map such that

$$
\beta\left(\dot{I}^{n+1}-\bigcup_{i=1}^{m} R_{i}\right)=\{s\} .
$$

If $S$ is $n$-simple, then

$$
\langle\beta\rangle=\sum_{i=1}^{m} \epsilon_{i}\left\langle\beta \circ \alpha_{i}\right\rangle
$$

where $\epsilon_{i}$ is the degree of the map 


$$
\alpha_{i}:\left(I^{n}, \dot{I}^{n}\right) \rightarrow\left(\dot{I}^{n+1}, \dot{I}^{n+1}-R_{i}\right)
$$

defined in terms of orientations consistent with the inclusions

$$
\left(I^{n}, \dot{I}^{n}\right) \subset\left(\dot{I}^{n+1}, J^{n}\right) \supset \dot{I}^{n+1} \subset\left(\dot{I}^{n+1}, \dot{I}^{n+1}-R_{i}\right) .
$$

4. Description of the exact couple $\nabla(K, Y, v)$. Suppose $K$ is a cell complex on the topological space $X$. By "cell complex" we mean essentially a "CW complex" or a "CW decomposition of $X$ " as described in [9] and [8], though we find it more convenient to regard the $n$-cells of $K$ not as subsets of $X$ but rather as continuous maps of the $n$-cube $I^{n}$ into $X$, the "characteristic maps." Then the $q$-skeleton $K_{q}$ is the union of the ranges of all cells with dimension $\leqq q$.

Suppose further that $Y$ is an arcwise connected topological space whose fundamental group is abelian and operates trivially on the higher homotopy groups, and suppose

$$
v: X \rightarrow Y
$$

is a continuous map. Let $v_{q}=v \mid K_{q}$ and let $U_{q}$ be the arcwise component of $v_{q}$ in $Y^{K_{q}}$, the space of all continuous maps of $K_{q}$ into $Y$ with the compactopen topology. The restriction operation defines fibre maps

$$
r_{q}: U_{q} \rightarrow U_{q-1}, \quad r_{q}(u)=u \mid K_{q-1} \text { for } u \in U_{q},
$$

in the sense of Serre [3], with the fibres

$$
F_{q}=r_{q}^{-1}\left\{v_{q-1}\right\}=U_{q} \cap\left\{u|u| K_{q-1}=v \mid K_{q-1}\right\},
$$

and with the corresponding exact homotopy sequences

$$
\begin{aligned}
& \cdots \rightarrow \pi_{p}\left(U_{q}, v_{q}\right) \rightarrow \pi_{p}\left(U_{q-1}, v_{q-1}\right) \rightarrow \pi_{p-1}\left(F_{q}, v_{q}\right) \rightarrow \pi_{p-1}\left(U_{q}, v_{q}\right) \rightarrow \cdots \\
& \cdots \rightarrow \pi_{1}\left(U_{q}, v_{q}\right) \rightarrow \pi_{1}\left(U_{q-1}, v_{q-1}\right) \rightarrow \pi_{0}\left(F_{q}, v_{q}\right) \rightarrow \pi_{0}\left(U_{q}, v_{q}\right)=\{0\} .
\end{aligned}
$$

It will be seen in the next two sections that the group $\pi_{1}\left(F_{q}, v_{q}\right)$ is abelian and that the set $\pi_{0}\left(F_{q}, v_{q}\right)$ can be given an abelian group structure with respect to which the boundary operator from $\pi_{1}\left(U_{q-1}, v_{q-1}\right)$ is a homomorphism. We also note that $K_{q}$ and $v_{q}$ are empty and $U_{q}=F_{q}=\left\{v_{q}\right\}$ in case $q<0$, and that $U_{0}=F_{0}$.

The exact couple $\nabla(K, Y, v)$ represented by the diagram

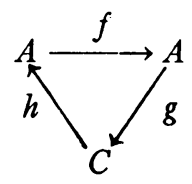

may now be defined as follows:

$$
A=\underset{p, q}{\oplus} A_{p, q} \text { and } C=\underset{p, q}{\oplus} C_{p, q}
$$


where the (weak) direct sums extend over all ordered pairs of integers $(p, q)$ and where

$$
\begin{gathered}
A_{p, q}=\pi_{p}\left(U_{q}, v_{q}\right) \text { and } C_{p, q}=\pi_{p}\left(F_{q}, v_{q}\right) \text { for } p \geqq 0, \\
A_{p, q}=C_{p, q}=\{0\} \text { for } p<0 .
\end{gathered}
$$

Furthermore

$$
f \mid A_{p, q}: A_{p, q} \rightarrow A_{p, q-1}
$$

is induced by $r_{q}$ if $p \geqq 0$ and is trivial if $p<0$;

$$
g \mid A_{p, q}: A_{p, q} \rightarrow C_{p-1, q+1}
$$

is the boundary operator associated with $r_{q+1}$ if $p \geqq 1$ and is trivial if $p<1$;

$$
h \mid C_{p, q}: C_{p, q} \rightarrow A_{p, q}
$$

is induced by the inclusion $F_{q} \subset U_{q}$ if $p \geqq 0$ and is trivial if $p<0$.

The above procedure of combining a sequence of related exact sequences into a single exact couple is the same as that followed by Massey [1] in several algebraically analogous situations.

Of course the graded structure of the exact couple $\nabla(K, Y, v)$ is inherited by its $n$th derived couple $\nabla^{(n)}(K, Y, v)$, which contains the exact sequences

$$
\cdots \rightarrow C_{p, q}^{(n)} \stackrel{h^{(n)}}{\longrightarrow} A_{p, q}^{(n)} \stackrel{f^{(n)}}{\longrightarrow} A_{p, q-1}^{(n)} \stackrel{g^{(n)}}{\longrightarrow} C_{p-1, q+n}^{(n)} \rightarrow \cdots
$$

and whose differential operator $d^{(n)}=g^{(n)} \circ h^{(n)}$ maps $C_{p, q}^{(n)}$ into $C_{p-1, q+n+1}^{(n)}$.

5. Representation of $C_{p, q}$ by cochains. Suppose $p \geqq 0$ and $q \geqq 0$. Since the elements of $C_{p, q}$ are the arcwise components of

$$
\left(F_{q}, v_{q}\right)\left(I^{p}, \dot{I}^{p}\right)
$$

and since this function space is a subspace of

$$
\left(Y^{K_{q}}\right)^{I^{p}}
$$

which is homeomorphic with

$$
Y^{I^{p} \times K_{q}},
$$

there corresponds to each $c \in C_{p, q}$ a homotopy class of continuous maps

$$
u: I^{p} \times K_{q} \rightarrow Y
$$

such that

$$
u(\xi, x)=v(x) \text { for }(\xi, x) \in \dot{I}^{p} \times K_{q} \cup I^{p} \times K_{q-1} .
$$

For each $q$-cell $\sigma: I^{q} \rightarrow X$ of $K$ we construct the map

$$
u_{\sigma}: \dot{I}^{p+q+1} \rightarrow Y
$$


by identifying $I^{p+q+1}$ with $I^{p} \times I^{q} \times I$ and setting

$$
\begin{aligned}
& u_{\sigma}(\xi, \eta, 0)=u[\xi, \sigma(\eta)] \text { for }(\xi, \eta, 0) \in I^{p+q}, \\
& u_{\sigma}(\xi, \eta, t)=v[\sigma(\eta)] \quad \text { for }(\xi, \eta, t) \in J^{p+q} .
\end{aligned}
$$

Note that under our identification $I^{p+q}$ and $J^{p+q}$ correspond to

$$
I^{p} \times I^{q} \times\{0\} \text { and } I^{p} \times I^{q} \times\{1\} \cup \dot{I}^{p} \times I^{q} \times I \cup I^{p} \times \dot{I}^{q} \times I
$$

respectively. Inasmuch as the element of $\pi_{p+q}(Y)$ represented by $u_{0}$ is the same for all maps $u$ representing $c$, we thus associate with $c$ a $q$-dimensional cochain $\gamma(c)$ of $K$ with coefficients in $\pi_{p+q}(Y) ; \gamma(c)(\sigma)$ is represented by $u_{\sigma}\left({ }^{4}\right)$.

The map

$$
\gamma: C_{p, q} \rightarrow C^{q}\left[K, \pi_{p+q}(Y)\right]
$$

is univalent. In fact let $c$ and $c^{\prime}$ be elements of $C_{p, q}$ represented by maps $u$ and $u^{\prime}$ such that $u_{\sigma}$ and $u_{\sigma}^{\prime}$ are homotopic for each $q$-cell $\sigma$ of $K$. Inasmuch as $u_{\sigma}$ and $u_{\sigma}^{\prime}$ agree on $J^{p+q}$ we can choose homotopies

$$
w_{\sigma}: \dot{I}^{p+q+1} \times I \rightarrow Y
$$

which deform $u_{\sigma}$ into $u_{\sigma}^{\prime}$ relative to $J^{p+q}$, and hence obtain a homotopy

$$
w: I^{p} \times K_{q} \times I \rightarrow Y
$$

which deforms $u$ into $u^{\prime}$ relative to $\dot{I}^{p} \times K_{q} \cup I^{p} \times K_{q-1}$ in such a way that

$$
w[\xi, \sigma(\eta), \lambda]=w_{\sigma}[(\xi, \eta, 0), \lambda]
$$

whenever $\xi \in I^{p}, \eta \in I^{q}, \lambda \in I$ and $\sigma$ is any $q$-cell of $K$. Therefore $c=c^{\prime}$.

Furthermore $\gamma$ is onto in case $p \geqq 1$. Given any $q$-dimensional cochain $\alpha$ of $K$ with coefficients in $\pi_{p+q}(Y)$ we may choose for each $q$-cell $\sigma$ of $K$ a continuous map

$$
\phi_{\sigma}: \dot{I}^{p+q+1} \rightarrow Y
$$

which represents $\alpha(\sigma)$ and for which

$$
\phi_{\sigma}(\xi, \eta, t)=v[\sigma(\eta)] \quad \text { whenever }(\xi, \eta, t) \in J^{p+q}
$$

We may then construct a continuous map

$$
\phi: I^{p} \times K_{q} \rightarrow Y
$$

such that

$$
\phi(\xi, x)=v(x) \text { for }(\xi, x) \in \dot{I}^{p} \times K_{q} \cup I^{p} \times K_{q-1}
$$

(4) While we shall derive our basic results directly without use of the theory of obstructions, we note that $\gamma(c)$ corresponds to a $p+q$ dimensional difference cochain on $I^{p} \times K_{q}$, separating $u$ and the map carrying $(\xi, x) \in I^{p} \times K_{q}$ into $v(x)$. 
and such that

$$
\phi[\xi, \sigma(\eta)]=\phi_{\sigma}(\xi, \eta, 0)
$$

whenever $\xi \in I^{p}, \eta \in I^{q}$ and $\sigma$ is any $q$-cell of $K$. The maps

$$
\psi_{\xi}: K_{q} \rightarrow Y, \quad \psi_{\xi}(x)=\phi(\xi, x) \text { for } x \in K_{q}
$$

corresponding to $\xi \in I^{p}$ are all homotopic to each other, with $\psi_{\xi}=v_{q}$ in case $\xi \in \dot{I}^{p}$ (which is nonempty because $p \geqq 1$ ), hence $\psi_{\xi} \in U_{q}$ for all $\xi \in I^{p}$. Since also $\psi_{\xi} \mid K_{q-1}=v_{q-1}$ whenever $\xi \in I^{p}$, the map $\phi$ represents an element $c$ of $C_{p, q}$, and clearly $\gamma(c)=\alpha$.

It is sometimes convenient to know the following alternate description of $\gamma$ : Suppose an element $c$ of $C_{p, q}$ is represented by a map $u$ as above, $\sigma$ is a q-cell of $K$, and suppose

$$
s_{\lambda}: I^{p+q} \rightarrow Y
$$

corresponding to $\lambda \in I$ are maps forming a homotopy such that

$$
\begin{aligned}
& s_{1}(\xi, \eta)=u[\xi, \sigma(\eta)] \text { for }(\xi, \eta) \in I^{p+q}, \\
& s_{\lambda}(\xi, \eta)=v[\sigma(\lambda \eta)] \text { for } \lambda \in I \text { and }(\xi, \eta) \in \dot{I}^{p+q} .
\end{aligned}
$$

Then $\gamma(c)(\sigma)$ is the homotopy class of the map

$$
s_{0}:\left(I^{p+q}, \dot{I}^{p+q}\right) \rightarrow(Y, v[\sigma(\theta)]) .
$$

To prove this assertion we simply extend the maps $s_{\lambda}$ to maps

$$
S_{\lambda}: \dot{I}^{p+q+1} \rightarrow Y
$$

by letting

$$
S_{\lambda}(\xi, \eta, t)=v[\sigma(\lambda \eta)] \text { for } \lambda \in I \text { and }(\xi, \eta, t) \in J^{p+q} \text {. }
$$

Since $S_{1}=u_{\sigma}$ and $S_{0}\left(J^{p+q}\right)=\{v[\sigma(\theta)]\}$, the three maps $u_{\sigma}, S_{0}$ and $s_{0}$ all represent $\gamma(c)(\sigma)$.

Using the second characterization of $\gamma$ one may easily verify that $\gamma$ is a homomorphism in case $p \geqq 1$. It follows that $C_{1, q}$ is abelian.

6 . The homomorphisms $g$ and $\gamma \circ g$. Suppose $p \geqq 1$ and $q \geqq 0$. To each $a \in A_{p, q}$ corresponds a homotopy class of continuous maps

$$
w: I^{p} \times K_{q} \rightarrow Y
$$

such that

$$
w(\xi, x)=v(x) \text { for }(\xi, x) \in \dot{I}^{p} \times K_{q} .
$$

In order to find $g(a) \in C_{p-1, q+1}$ we apply the definition of the boundary operator associated with the fibre map $r_{q+1}$ and extend $w$ to a continuous map

$$
W: I^{p} \times K_{q+1} \rightarrow Y
$$


such that

$$
W(\xi, x)=v(x) \text { for }(\xi, x) \in J^{p-1} \times K_{q+1} .
$$

Then $g(a)$ is represented by the map

$$
u=W \mid I^{p-1} \times K_{q+1} \text {. }
$$

While this representation involves the arbitrary extension $W$ of $w$, we shall presently express the cochain

$$
\gamma[g(a)] \in C^{q+1}\left[K, \pi_{p+q}(Y)\right]
$$

directly in terms of $w$.

Let $\sigma: I^{q+1} \rightarrow X$ be a $q+1$ cell of $K$. We identify $I^{p+q+1}$ with $I^{p} \times I^{q+1}$ and choose maps

$$
S_{\lambda}: I^{p+q+1} \rightarrow Y
$$

corresponding to $\lambda \in I$ and forming a homotopy such that

$$
\begin{aligned}
& S_{1}(\xi, \eta)=W[\xi, \sigma(\eta)] \text { for }(\xi, \eta) \in I^{p} \times I^{q+1}, \\
& S_{\lambda}(\xi, \eta)=v[\sigma(\lambda \eta)] \quad \text { for } \lambda \in I \text { and }(\xi, \eta) \in \dot{I}^{p} \times \dot{I}^{q+1} \cup J^{p-1} \times I^{q+1} .
\end{aligned}
$$

Now $\dot{I}^{p+q+1}$ is the union of the two $p+q$ cells

$$
M=I^{p-1} \times I^{q+1} \text { and } N=I^{p} \times \dot{I}^{q+1} \cup J^{p-1} \times I^{q+1}
$$

whose intersection

$$
M \cap N=I^{p-1} \times \dot{I}^{q+1} \cup \dot{I}^{p-1} \times I^{q+1}
$$

is mapped by $S_{0}$ onto the point $v[\sigma(\theta)]$. Defining the maps $\mu$ and $\nu$ of $\dot{I}^{p+q+1}$ into $Y$ by the formulae

$$
\begin{aligned}
\mu(\zeta) & =S_{0}(\zeta) \text { for } \zeta \in M, & & \mu(\zeta)=v[\sigma(\theta)] \text { for } \zeta \in N, \\
\nu(\zeta) & =S_{0}(\zeta) \text { for } \zeta \in N, & \nu(\zeta) & =v[\sigma(\theta)] \text { for } \zeta \in M,
\end{aligned}
$$

and noting that $S_{0} \mid \dot{I}^{p+q+1}$ is inessential, we find that $\langle\mu\rangle+\langle\nu\rangle=0$. Furthermore the map

$$
\begin{gathered}
e:\left(I^{p+q}, \dot{I}^{p+q}\right) \rightarrow\left(\dot{I}^{p+q+1}, N\right), \\
e(\zeta)=\left(\zeta_{1}, \cdots, \zeta_{p-1}, 0, \zeta_{p}, \cdots, \zeta_{p+q}\right) \text { for } \zeta \in I^{p+q}
\end{gathered}
$$

yields maps

$$
s_{\lambda}=S_{\lambda} \circ e: I^{p+q} \rightarrow Y \text { for } \lambda \in I
$$

which form a homotopy such that

$$
\begin{aligned}
& s_{1}(\zeta)=u\left[\zeta_{1}, \cdots, \zeta_{p-1}, \sigma\left(\zeta_{p}, \cdots, \zeta_{p+q}\right)\right] \text { for } \zeta \in I^{p+q} \\
& s_{\lambda}(\zeta)=v\left[\sigma\left(\lambda \zeta_{p}, \cdots, \lambda \zeta_{p+q}\right)\right] \text { for } \lambda \in I \text { and } \zeta \in \dot{I}^{p+q}
\end{aligned}
$$


and $s_{0}=\mu \circ e$. Applying the second description of $\gamma$ in the preceding section, and observing that $e$ has degree $(-1)^{q+1}$, we see that

$$
\langle\mu\rangle=(-1)^{a+1} \gamma[g(a)] .
$$

Finally we consider for $\lambda \in I$ the maps

$$
\begin{aligned}
& t_{\lambda}: \dot{I}^{p+q+1} \rightarrow Y, \quad t_{\lambda}\left|N=S_{\lambda}\right| N, \\
& t_{\lambda}(\xi, \eta)=v[\sigma(\lambda \eta)] \text { for }(\xi, \eta) \in M,
\end{aligned}
$$

which form a homotopy such that $t_{0}=\nu$ and

$$
\begin{array}{ll}
t_{1}(\xi, \eta)=w(\xi, \sigma(\eta)) & \text { for }(\xi, \eta) \in I^{p} \times \dot{I}^{q+1}, \\
t_{1}(\xi, \eta)=v[\sigma(\eta)] & \text { for }(\xi, \eta) \in \dot{I}^{p} \times I^{q+1} .
\end{array}
$$

We conclude that $\left(^{5}\right)$

$$
\gamma[g(a)](\sigma)=(-1)^{q+1}\langle\mu\rangle=(-1)^{q}\langle\nu\rangle=(-1)^{q}\left\langle t_{1}\right\rangle .
$$

Using this result one may easily verify that the map

$$
\gamma \circ g: A_{p, q} \rightarrow C^{q+1}\left[K, \pi_{p+q}(Y)\right]
$$

is a homomorphism. Of course we already know this for $p>1$, because in this case the boundary operator $g$ associated with $r_{q+1}$ is a homomorphism and $\gamma$ is a homomorphism as a consequence of the preceding section. Now however, after verification that $\gamma \circ g$ is a homomorphism also for $p=1$, we note that the set

$$
\gamma\left(C_{0, q+1}\right)=\gamma\left[g\left(A_{1, q}\right)\right]
$$

is a subgroup of the abelian group

$$
C^{q+1}\left[K, \pi_{q+1}(Y)\right]
$$

recall from the preceding section that $\gamma$ is univalent, and give $C_{0, q+1}$ a group structure by requiring that $\gamma$ shall be an isomorphism. Then, since $\gamma \circ g$ is a homomorphism, so is $g=\gamma^{-1} \circ(\gamma \circ g)$.

7. The differential operator $d^{(0)}=g \circ h$. For $p \geqq 1$ and $q \geqq 0$ we shall establish commutativity of the diagram

$$
\begin{aligned}
& C_{p, q} \stackrel{d^{(0)}=g \circ h}{\longrightarrow} C_{p-1, q+1} \\
& \gamma \downarrow \quad \downarrow \gamma \\
& C^{q}\left[K, \pi_{p+q}(Y)\right] \underset{(-1)^{q} \delta}{\longrightarrow} C^{q+1}\left[K, \pi_{p+q}(Y)\right]
\end{aligned}
$$

(5) We note (see footnote 4) that $(\gamma \circ g)(a)$ corresponds to a $p+q+1$ dimensional obstruction cochain on $\dot{I}^{p} \times K_{q+1}$, associated with the extension of $w$ to $I^{p} \times K_{q} \cup I^{p} \times K_{q+1}$ carrying $(\xi, x)$ $\in I^{p} \times K_{q+1}$ into $v(x)$. 
where $\delta$ is the coboundary operator, provided the complex $K$ satisfies the following condition $\left({ }^{\circ}\right)$ :

If $\sigma: I^{a+1} \rightarrow X$ is a $q+1$ cell of $K$ and if $\Phi$ is the set of all those continuous maps $\phi: I^{q} \rightarrow \dot{I}^{q+1}$ for which the map $\sigma \circ \phi: I^{q} \rightarrow X$ is a $q$ cell of $K$, then

$$
\dot{I}^{q+1}-\sigma^{-1}\left(K_{q-1}\right) \subset \bigcup_{\phi \in \Phi} \phi\left(I^{q}-\dot{I}^{q}\right) .
$$

We observe that in any event the sets $\phi\left(I^{q}-\dot{I}^{q}\right)$ corresponding to distinct elements $\phi$ of $\Phi$ are disjoint, and $\Phi$ is finite. Our condition implies (but is not equivalent to) the statement that all $q$-dimensional faces of $\sigma$ in $K$ are of the type $\sigma \circ \phi$ for some (not necessarily unique) element $\phi$ of $\Phi$. The condition holds for all the classical examples of cell complexes for which incidence matrices can be computed explicitly. Moreover one may associate in well known fashion with every cell complex another complex of the same homotopy type and satisfying the above condition.

Suppose $q \geqq 1, c \in C_{p, q}$ and $\sigma: I^{q+1} \rightarrow X$ is a $q+1$ cell of $K$.

Let $u$ be a map representing $c$, as in $\S 5$. Then $u$ also represents $h(c) \in A_{p, q}$, and $\S 6$ applies with $a=h(c)$ and $w=u$. Accordingly

$$
\gamma\left[d^{(0)}(c)\right](\sigma)=(-1)^{q}\langle t\rangle
$$

where

$$
\begin{gathered}
t: \dot{I}^{p+q+1} \rightarrow Y, \\
t(\xi, \eta)=u[\xi, \sigma(\eta)] \text { for }(\xi, \eta) \in I^{p} \times \dot{I}^{q+1}, \\
t(\xi, \eta)=v[\sigma(\eta)] \quad \text { for }(\xi, \eta) \in \dot{I}^{p} \times I^{q+1} .
\end{gathered}
$$

Here $I^{p+q+1}$ is identified with $I^{p} \times I^{q+1}$. Letting

$$
D=\dot{I}^{p} \times I^{q+1} \cup I^{p} \times \sigma^{-1}\left(K_{q-1}\right)
$$

we note that

$$
t(\xi, \eta)=v[\sigma(\eta)] \text { for }(\xi, \eta) \in D
$$

and choose maps

$$
T_{\lambda}: \dot{I}^{p+q+1} \rightarrow Y \text { for } \lambda \in I
$$

which form a homotopy such that $T_{1}=t$ and

$$
T_{\lambda}(\xi, \eta)=v[\sigma(\lambda \eta)] \text { for } \lambda \in I \text { and }(\xi, \eta) \in D \text {. }
$$

Defining $\Phi$ as above we associate with each $\phi \in \Phi$ the map

(6) This restriction is not essential. One may verify that all the homomorphisms in the diagram are natural with respect to the category of cell complexes and cellular maps, and then one needs to verify commutativity only in the special case in which $K$ is the usual cell complex on $I^{q+1}$. Alternately one may use the corresponding result of obstruction theory (Theorem 2.7.4 of [8]), in view of the connection indicated in footnotes 4 and 5 . 


$$
\begin{gathered}
\phi: I^{p+q} \rightarrow \dot{I}^{p+q+1}, \\
\phi(\xi, \eta)=(\xi, \phi(\eta)) \text { for }(\xi, \eta) \in I^{p} \times I^{q},
\end{gathered}
$$

and note that the two maps

$$
\begin{aligned}
\phi:\left(I^{q}, \dot{I}^{q}\right) & \rightarrow\left(\dot{I}^{q+1}, \dot{I}^{q+1}-\phi\left(I^{q}-\dot{I}^{q}\right)\right), \\
\Phi:\left(I^{p+q}, \dot{I}^{p+q}\right) & \rightarrow\left(\dot{I}^{p+q+1}, \dot{I}^{p+q+1}-\phi\left(I^{p+q}-\dot{I}^{p+q}\right)\right)
\end{aligned}
$$

have the same degree. Inasmuch as $T_{0}$ maps the set

$$
\dot{I}^{p+q+1}-\bigcup_{\phi \in \Phi} \Phi\left(I^{p+q}-I^{p+q}\right) \subset D
$$

onto the single point $v[\sigma(\theta)]$, it follows from the homotopy addition theorem stated in $\$ 3$ that

$$
\langle t\rangle=\left\langle T_{0}\right\rangle=\sum_{\phi \in \Phi} \text { degree }(\phi) \cdot\left\langle T_{0} \circ \bar{\phi}\right\rangle .
$$

For each $\phi \in \Phi$ the maps $T_{\lambda} \circ \bar{\phi}: I^{p+q} \rightarrow Y$ corresponding to $\lambda \in I$ form a homotopy such that

$$
\begin{aligned}
& \left(T_{1} \circ \bar{\phi}\right)(\zeta)=u\left[\left(\zeta_{1}, \cdots, \zeta_{p}\right),(\sigma \circ \phi)\left(\zeta_{p+1}, \cdots, \zeta_{p+q}\right)\right] \text { for } \zeta \in I^{p+q} \\
& \left(T_{\lambda} \circ \bar{\phi}\right)(\zeta)=(v \circ \sigma)\left[\lambda \cdot \phi\left(\zeta_{p+1}, \cdots, \zeta_{p+q}\right)\right] \text { for } \lambda \in I \text { and } \zeta \in \dot{I}^{p+q} .
\end{aligned}
$$

We extend $T_{\lambda} \circ \Phi$ to $W_{\lambda}: \dot{I}^{p+q+1} \rightarrow Y$ by setting

$$
W_{\lambda}(\zeta)=(v \circ \sigma)\left[\lambda \cdot \phi\left(\zeta_{p+1}, \cdots, \zeta_{p+q}\right)\right] \text { for } \lambda \in I \text { and } \zeta \in J^{p+q}
$$

and thereby obtain a homotopy joining $W_{1}=u_{\sigma \circ \phi}$ to the extension $W_{0}$ of $T_{0} \circ \Phi$, with $W_{0}\left(J^{p+q}\right)=\{(v \circ \sigma)(\theta)\}$, whence

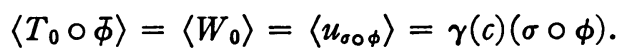

Combining these results we find that

$$
\left(\gamma \circ d^{(0)}\right)(c)(\sigma)=(-1)^{q} \sum_{\phi \in \Phi} \text { degree }(\phi) \cdot \gamma(c)(\sigma \circ \phi) .
$$

Now suppose the cell complex $K$ is oriented as follows: For $n=0,1,2, \ldots$. let $\omega_{n}$ be a generator of $H_{n}\left(I^{n}, \dot{I}^{n}\right)$ such that if $n \geqq 1$ then $\omega_{n}$ is mapped onto $\omega_{n-1}$ by the canonical isomorphisms

$$
H_{n}\left(I^{n}, \dot{I}^{n}\right) \simeq H_{n-1}\left(\dot{I}^{n}, J^{n-1}\right) \simeq H^{n-1}\left(I^{n-1}, \dot{I}^{n-1}\right) .
$$

Then orient the chain group $C_{n}(K)=H_{n}\left(K_{n}, K_{n-1}\right)$ by choosing as generators the elements $\tau_{*}\left(\omega_{n}\right)$ corresponding to the $n$-cells $\tau:\left(I^{n}, \dot{I}^{n}\right) \rightarrow\left(K_{n}, K_{n-1}\right)$ of $K$.

It readily follows from these conventions that

$$
\partial\left[\sigma_{*}\left(\omega_{q+1}\right)\right]=\sum_{\phi \in \Phi} \text { degree }(\phi) \cdot(\sigma \circ \phi)_{*}\left(\omega_{q}\right),
$$

and hence that 


$$
\delta[\gamma(c)](\sigma)=\sum_{\phi \in \Phi} \text { degree }(\phi) \cdot \gamma(c)(\sigma \circ \phi) .
$$

Thus $\left(\gamma \circ d^{(0)}\right)(c)(\sigma)=(-1)^{q}(\delta \circ \gamma)(c)(\sigma)$, as was to be shown.

For $q=0$ certain parts of the preceding argument would be meaningless, but one may follow it up to the definition of $T_{\lambda}$ and replace the remainder by much simpler reasoning.

We conclude that for $p \geqq 0$ and $q \geqq 0$ the chain transformation $\gamma$ induces isomorphisms

$$
\gamma^{*}: C_{p, q}^{(1)} \rightarrow B^{q}\left[K, \pi_{p+q}(Y)\right],
$$

which are onto in case $p \geqq 1$.

The result just obtained gives only a partial description of the groups $C_{0, q}^{(1)}$, but this does not really matter because $C_{0, q}^{(\infty)}=\{0\}$. In fact one may verify quite directly that $d^{(q-1)}\left(C_{1,0}^{(q-1)}\right)=C_{0, q}^{(q-1)}$.

8. A normal chain for $\pi_{p}\left(Y^{X}, v\right)$. Suppose $p \geqq 1$. The restriction operation defines continuous maps

$$
\rho_{q}:\left(Y^{\mathbf{X}}, v\right) \rightarrow\left(Y^{K_{q}}, v_{q}\right), \quad \rho_{q}(u)=u \mid K_{q} \text { for } u \in Y^{\mathbf{X}},
$$

carrying the arcwise component of $v$ in $Y^{\boldsymbol{x}}$ into $U_{q}$. Letting $G_{p, q}$ be the kernel of the induced homomorphism

$$
\rho_{q *}: \pi_{p}\left(Y^{\mathbf{X}}, v\right) \rightarrow A_{p, q}
$$

we obtain the normal chain

$$
\pi_{p}\left(Y^{X}, v\right)=G_{p,-1} \supset G_{p, 0} \supset G_{p, 1} \supset G_{p, 2} \supset \cdots .
$$

Assuming now that $\operatorname{dim} X=Q<\infty$, so that the chain descends in $Q+1$ steps to $G_{p, Q}=\{0\}$, we shall prove that

$$
G_{p, q-1} / G_{p, q} \simeq C_{p, q}^{(n)} \text { for } n \geqq \max (q, Q-q) .
$$

In fact if $n \geqq Q-q$, then

$$
\begin{aligned}
K_{q+n} & =X, \quad A_{p, q+n}=\pi_{p}\left(Y^{X}, v\right), \quad f^{n} \mid A_{p, q+n}=\rho_{q *}, \\
A_{p, q}^{(n)} & =f^{n}\left(A_{p, q+n}\right)=\text { image } \rho_{q *},
\end{aligned}
$$

and we see from the commutative diagram

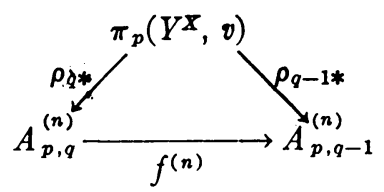

that $G_{p, q-1} / G_{p, q} \simeq \rho_{q *}\left(G_{p, q-1}\right)=$ kernel $\left(f^{(n)} \mid A_{p, q}^{(n)}\right)$. Furthermore the exact couple $\nabla^{(n)}(K, Y, v)$ contains in case $n \geqq q$ the exact sequence 


$$
\{0\}=A_{p+1, q-n-1}^{(n)} \stackrel{g^{(n)}}{\longrightarrow} C_{p, q}^{(n)} \stackrel{h^{(n)}}{\longrightarrow} A_{p, q}^{(n)} \stackrel{f^{(n)}}{\longrightarrow} A_{p, q-1}^{(n)}
$$

which shows that kernel $\left(f^{(n)} \mid A_{p, q}^{(n)}\right) \simeq C_{p, q}^{(n)}$.

In $\S 11$ we shall construct examples which show that $\pi_{1}\left(Y^{X}, v\right)$ can be nonabelian, in spite of our assumption that $Y$ is simple in all dimensions. However, since the groups $C_{1, q}$ are abelian, the above normal chain shows that $\pi_{1}\left(Y^{x}, v\right)$ is solvable. Actually the following stronger statement is true: $\pi_{1}\left(Y^{x}, v\right)$ is nilpotent and, more generally, for every $p \geqq 1$ the group $\pi_{p}\left(Y^{\mathbb{X}}, v\right)$ is nilpotent with respect to the operation of $\pi_{1}\left(Y^{X}, v\right)$. We shall in fact verify presently that

$$
\left[\pi_{1}\left(Y^{X}, v\right), G_{p, q-1}\right] \subset G_{p, q}
$$

where [,] is the Whitehead product.

Assuming that $a \in \pi_{1}\left(Y^{x}, v\right)$ and $a^{\prime} \in G_{p, q-1}$ we may represent $a$ and $a^{\prime}$ by continuous maps

$$
u: I \times X \rightarrow Y \text { and } u^{\prime}: I^{p} \times X \rightarrow Y
$$

such that

$$
\begin{aligned}
u(t, x) & =v(x) \text { for }(t, x) \in \dot{I} \times X, \\
u^{\prime}(\xi, x) & =v(x) \text { for }(\xi, x) \in \dot{I}^{p} \times X \cup I^{p} \times K_{q-1} .
\end{aligned}
$$

Then $\left[a, a^{\prime}\right] \in \pi_{p}\left(Y^{x}, v\right)$ is represented by the continuous map

$$
\begin{gathered}
w: \dot{I}^{1+p} \times X \rightarrow Y, \\
w(t, \xi, x)=u(t, x) \text { for }(t, \xi, x) \in I \times \dot{I}^{p} \times X, \\
w(t, \xi, x)=u^{\prime}(\xi, x) \text { for }(t, \xi, x) \in \dot{I} \times I^{p} \times X,
\end{gathered}
$$

and in order to prove that $\left[a, a^{\prime}\right] \in G_{p, q}$ it is clearly sufficient to show that $w \mid \dot{I}^{1+p} \times K_{q}$ can be continuously extended over $I^{p+1} \times K_{q}$. For this purpose we first construct the extension

$$
\begin{aligned}
W: \dot{I}^{1+p} & \times K_{q} \cup I^{1+p} \times K_{q-1} \rightarrow Y, \\
W(t, \xi, x) & =u(t, x) \text { for }(t, \xi, x) \in I \times I^{p} \times K_{q-1},
\end{aligned}
$$

and complete our argument by verifying that for each $q$-cell $\sigma$ of $K$ the map

$$
\begin{gathered}
W_{\sigma}: \dot{I}^{1+p+q} \rightarrow Y, \\
W_{\sigma}(t, \xi, \eta)=W(t, \xi, \sigma(\eta)) \text { for }(t, \xi, \eta) \in \dot{I}^{1+p+q},
\end{gathered}
$$

is inessential. We choose continuous maps

$$
s_{\lambda}: I^{p+q} \rightarrow Y \text { for } \lambda \in I
$$

forming a homotopy such that

$$
\begin{aligned}
& s_{1}(\xi, \eta)=u^{\prime}(\xi, \sigma(\eta)) \text { for }(\xi, \eta) \in I^{p+q} \\
& s_{\lambda}(\xi, \eta)=(v \circ \sigma)(\lambda \eta) \text { for } \lambda \in I \text { and }(\xi, \eta) \in \dot{I}^{p+q} .
\end{aligned}
$$


Observing that

$$
u(t, \sigma(\lambda \eta))=(v \circ \sigma)(\lambda \eta) \text { for } \lambda \in I \text { and }(t, \eta) \in \dot{I} \times I^{q},
$$

we then construct the homotopy formed by the maps

$$
\begin{array}{ll}
\phi_{\lambda}: \dot{I}^{1+p+q} \rightarrow Y & \text { for } \quad \lambda \in I, \\
\phi_{\lambda}(t, \xi, \eta)=u(t, \sigma(\lambda \eta)) & \text { for }(t, \xi, \eta) \in I \times \dot{I}^{p+q}, \\
\phi_{\lambda}(t, \xi, \eta)=s_{\lambda}(\xi, \eta) & \text { for }(t, \xi, \eta) \in I \times I^{p+q} .
\end{array}
$$

Since $\phi_{1}=W_{\sigma}$ and $\left\langle\phi_{0}\right\rangle=\left[\langle\psi\rangle,\left\langle s_{0}\right\rangle\right]$, where

$$
\psi:(I, \dot{I}) \rightarrow(Y,(v \circ \sigma)(\theta)), \quad \psi(t)=u(t, \sigma(\theta)) \text { for } t \in I,
$$

the $p+q$ simplicity of $Y$ implies that $\left\langle\phi_{0}\right\rangle=0$, hence $\left\langle W_{\sigma}\right\rangle=0$.

Of course the above normal chain leads immediately to some qualitative results concerning the homotopy groups of $Y^{X}$. For example if the homotopy groups of $Y$ and the integral cohomology groups of $X$ all belong to a class of abelian groups satisfying the axioms $(\mathrm{I})$ and $\left(\mathrm{II}_{A}\right)$ of Serre [4], then the groups $\pi_{p}\left(Y^{\mathbb{X}}, v\right)$ belong to the same class for all $p \geqq 2$.

9. Invariance properties. For every fibre map $T: E \rightarrow B$ the homotopy sequences

$$
\cdots \rightarrow \pi_{p}\left(T^{-1}\{T(e)\}, e\right) \rightarrow \pi_{p}(E, e) \rightarrow \pi_{p}(B, T(e)) \rightarrow \cdots
$$

corresponding to the various points $e \in E$ form in a natural way a local system of exact sequences on the space $E$. Applying this principle simultaneously to all the fibre maps $r_{q}$, one finds that for fixed $K, X, Y$ the exact couples $\nabla(K, Y, v)$ corresponding to the various points $v \in Y^{\boldsymbol{x}}$ form a local system on the space $Y^{\boldsymbol{x}}$. This local system induces further local systems formed by all the derived couples $\nabla^{(n)}(K, Y, v)$. Of course this implies that the isomorphism type of $\nabla(K, Y, v)$ is determined by the homotopy class of $v$.

One may verify quite directly that the diagram

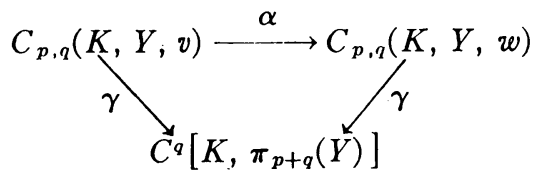

is commutative whenever $\alpha$ is the isomorphism induced by any path from $v$ to $w$ in $Y^{x}$. Therefore the local systems formed by the groups $C_{p, q}(K, Y, v)$ are simple. On the other hand the systems formed by the groups $A_{p, q}(K, Y, v)$ need not be simple, as shown by examples constructed in $\$ 11$.

If $\bar{K}$ is another cell complex on a space $\bar{X}$, if $\bar{v} \in Y^{\bar{X}}$, and if $\phi$ is a cellular map of $X$ into $\bar{X}$ with respect to $K$ and $\bar{K}$, which means that $\phi$ is continuous and $\phi\left(K_{q}\right) \subset \bar{K}_{q}$ for all $q$, then $\phi$ induces in obvious fashion a homomorphism

$$
\phi^{\dagger}: \nabla(\bar{K}, Y, \bar{v}) \rightarrow \nabla(K, Y, \bar{v} \circ \phi)
$$


which in turn induces homomorphisms

$$
\phi^{f(n)}: \nabla^{(n)}(\bar{K}, Y, \bar{v}) \rightarrow \nabla^{(n)}(K, Y, \bar{v} \circ \phi)
$$

of all the derived couples. We shall prove that in case $\phi$ is a homotopy equivalence $\phi^{*(1)}$ is an isomorphism onto.

First we verify that to every homotopy $\Phi: X \times I \rightarrow \bar{X}$ joining two cellular maps $\phi_{0}$ and $\phi_{1}$ correspond commutative diagrams

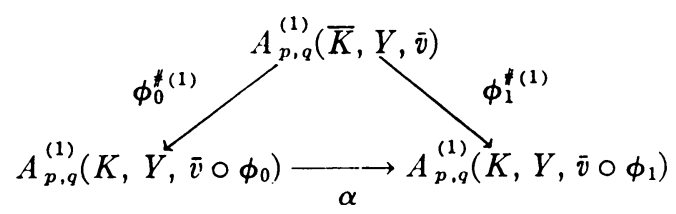

where $\alpha$ is the isomorphism induced by the curve

$$
V: I \rightarrow Y^{\boldsymbol{X}}, \quad V(t)(x)=(\bar{v} \circ \Phi)(x, t) \text { for } t \in I \text { and } x \in X .
$$

Since every homotopy can be deformed into a cellular homotopy, with the same $\alpha$, we may assume that $\Phi$ is cellular, which means that $\Phi\left(K_{q} \times I\right) \subset \bar{K}_{q+1}$ for all $q$. Assuming that $a \in A_{p, q}^{(1)}(\bar{K}, Y, \bar{v})$ we choose a continuous map

$$
u: I^{p} \times \bar{K}_{q+1} \rightarrow Y
$$

for which

$$
u(\xi, x)=\bar{v}(x) \text { for }(\xi, x) \in \dot{I}^{p} \times \bar{K}_{q+1}
$$

and such that $u \mid I^{p} \times \bar{K}_{q}$ represents $a$. The continuous maps

$$
w_{t}: I^{p} \times K_{q} \rightarrow Y, w_{t}(\xi, x)=u[\xi, \Phi(x, t)] \text { for }(\xi, x) \in I^{p} \times K_{q}
$$

corresponding to $t \in I$ form a homotopy such that $w_{0}$ represents $\phi_{0}^{*}(a), w_{1}$ represents $\phi_{1}^{\sharp}(a)$ and

$$
w_{t}(\xi, x)=V(t)(x) \text { for }(\xi, x) \in \dot{I}^{p} \times K_{q} .
$$

Accordingly $\alpha\left[\phi_{0}^{*}(a)\right]=\phi_{1}^{*}(a)$.

From this we infer that if $\bar{K}=K, \bar{X}=X$ and $\phi: X \rightarrow X$ is a cellular map homotopic to the identity map of $X$, then $\phi^{\prime(1)}$ is an isomorphism onto. In fact the homomorphisms

$$
\phi^{f(1)}: A_{p, q}^{(1)}(K, Y, \bar{v}) \rightarrow A_{p, q}^{(1)}(K, Y, \bar{v} \circ \phi)
$$

are in this case the isomorphisms onto induced by a certain curve from $\bar{v}$ to $\bar{v} \circ \phi$ in $Y^{x}$, and the five-lemma implies that the homomorphisms

$$
\phi^{f(1)}: C_{p, q}^{(1)}(K, Y, \bar{v}) \rightarrow C_{p, q}^{(1)}(K, Y, \bar{v} \circ \phi)
$$

are also isomorphisms onto. 
Now we are ready to prove our assertion that the homomorphism $\phi^{*(1)}$ induced by a homotopy equivalence $\phi: X \rightarrow \bar{X}$ is an isomorphism onto. Let $\psi: \bar{X} \rightarrow X$ be a cellular homotopy inverse of $\phi$. Since $\phi \circ \psi$ is homotopic to the identity map of $\bar{X}$, with $(\phi \circ \psi)^{*}=\psi^{*} \circ \phi^{*}$, the superposition of the two homomorphisms

$$
\nabla^{(1)}(\bar{K}, Y, \bar{v}) \stackrel{\phi^{*(1)}}{\longrightarrow} \nabla^{(1)}(K, Y, \bar{v} \circ \phi) \stackrel{\psi^{*(1)}}{\longrightarrow} \nabla^{(1)}(\bar{K}, Y, \bar{v} \circ \phi \circ \psi)
$$

is an isomorphism onto. Therefore $\phi^{\sharp(1)}$ is an isomorphism, and in order to show that $\phi^{i(1)}$ is onto it is sufficient to show that $\psi^{*(1)}$ is an isomorphism. However this follows from the fact that the superposition of the two homomorphisms

$$
\nabla^{(1)}(K, Y, \bar{v} \circ \phi) \stackrel{\psi^{*(1)}}{\longrightarrow} \nabla^{(1)}(\bar{K}, Y, \bar{v} \circ \phi \circ \psi) \stackrel{\phi^{f(1)}}{\longrightarrow} \nabla^{(1)}(K, Y, \bar{v} \circ \phi \circ \psi \circ \phi)
$$

is an isomorphism onto, because $\psi \circ \phi$ is homotopic to the identity map of $X$.

In view of the facts just established, and noting also the rather trivial effect of replacing $Y$ by another space of the same homotopy type, we conclude that the first derived couple $\nabla^{(1)}(K, Y, v)$ is an invariant of the homotopy type of $(X, Y, v)$. It follows of course that the same is true for all the higher derived couples.

10. Two examples. Here we indicate how our method operates in two relatively simple special cases. While these particular results are not new, our method led to their independent rediscovery, and we consider them interesting illustrations. Later, in $\$ 14$, we shall carry out the solution of a more original example.

First suppose $\Pi$ is an abelian group, $m \geqq 1, \pi_{m}(Y) \simeq \Pi, \pi_{i}(Y)=\{0\}$ for $i \neq m, X$ is a finite dimensional cellular space, and $v \in Y^{x}$. Then $C_{p, q}^{(1)}=\{0\}$ for $p+q \neq m, C_{p, m-p}^{(1)} \simeq H^{m-p}(X, \Pi), d^{(n)}$ is trivial for $n \geqq 1$, hence

$$
\pi_{p}\left(Y^{X}, v\right) \simeq H^{m-p}(X, \Pi) \text { for } p \geqq 1 .
$$

This result has also been obtained by A. Heller and by R. Thom, according to a letter from N. Steenrod.

Next suppose $X$ is an $m$-sphere, $m \geqq 1, Y$ is any simple space, and $v \in Y^{X}$. Let $K$ be a complex with one vertex $s \in X$ and one $m$-cell $\sigma:\left(I^{m}, \dot{I}^{m}\right) \rightarrow(X, s)$. Then $C_{p, q}=\{0\}$ unless $q=0$ or $q=m, C_{p, 0} \simeq \pi_{p}(Y), C_{p, m} \simeq \pi_{p+m}(Y)$, and the only nontrivial differential operator is $d^{(m-1)}: C_{p, 0} \rightarrow C_{p-1, m}$, which may be interpreted as follows: If

$$
\phi:\left(I^{p}, \dot{I}^{p}\right) \rightarrow(Y, v(s))
$$

is a continuous map, then to $\langle\phi\rangle \in \pi_{p}(Y, v(s))$ corresponds $c \in C_{p, 0}$ represented by

$$
u: I^{p} \times\{s\} \rightarrow Y, \quad u(\xi, s)=\phi(\xi) \text { for } \xi \in I^{p}
$$


Since $K_{m-1}=K_{0}, \quad u$ also represents an element $a \in A_{p, m-1}$ such that $f^{m-1}(a)=h(c)$. To $g(a)=d^{(m-1)}(c) \in C_{p-1, m}$ corresponds, according to $\S 6$, $(-1)^{m-1}\langle t\rangle \in \pi_{p-1+m}(Y)$, where

$$
\begin{gathered}
t: \dot{I}^{p+m} \rightarrow Y, \\
t(\xi, \eta)=u(\xi, \sigma(\eta))=\phi(\xi) \text { for }(\xi, \eta) \in I^{p} \times \dot{I}^{m}, \\
t(\xi, \eta)=(v \circ \sigma)(\eta) \quad \text { for }(\xi, \eta) \in \dot{I}^{p} \times I^{m} .
\end{gathered}
$$

Inasmuch as $\langle t\rangle=[\langle\phi\rangle,\langle v \circ \sigma\rangle]$, we have thus identified $d^{(m-1)}$ with Whitehead multiplication by $(-1)^{m-1}\langle v \circ \sigma\rangle$. Therefore the exact sequence

$$
\{0\} \rightarrow C_{p, m}^{(m)} \rightarrow \pi_{p}\left(Y^{X}, v\right) \rightarrow C_{p, 0}^{(m)} \rightarrow\{0\}
$$

obtained from $\S 8$ leads to an exact sequence

$$
\pi_{p+1}(Y) \stackrel{V}{\rightarrow} \pi_{p+m}(Y) \rightarrow \pi_{p}\left(Y^{X}, v\right) \rightarrow \pi_{p}(Y) \stackrel{V}{\rightarrow} \pi_{p-1+m}(Y)
$$

where $V(\alpha)=[\alpha,\langle v \circ \sigma\rangle]$. This exact sequence was first obtained by G. W. Whitehead in [7], without the assumption of simplicity of $Y$, through direct study of the fibre map $r_{m}: Y^{x} \rightarrow Y^{\{s\}}$.

11. The nonsimplicity of $Y^{X}$. In order to construct examples showing that simplicity of $Y$ does not imply simplicity of $Y^{\boldsymbol{x}}$, we shall first prove a general proposition connecting Whitehead products in $Y$ with Whitehead products in $Y^{s^{m}}$, where $S^{m}$ is an $m$-sphere and where $Y$ need not be simple. No implicit assumptions of simplicity will be made in this section, which is independent of the rest of the paper. We shall prove:

$$
\text { If } y \in Y, v: S^{m} \rightarrow\{y\}, p \geqq 1, n \geqq 1, m \geqq 1 \text { and }\left[\pi_{p}(Y, y), \pi_{n+m}(Y, y)\right] \neq 0 \text {, }
$$
then

$$
\left\lfloor\pi_{p}\left(Y^{s^{m}}, v\right), \pi_{n}\left(Y^{s^{m}}, v\right)\right] \neq 0 .
$$

In fact suppose

$$
\phi:\left(I^{p}, \dot{I}^{p}\right) \rightarrow(Y, y) \quad \text { and } \quad \psi:\left(I^{n+m}, \dot{I}^{n+m}\right) \rightarrow(Y, y)
$$

are continuous maps such that $[\langle\phi\rangle,\langle\psi\rangle] \neq 0$. Choosing $s \in S^{m}$ and a continuous map $\sigma:\left(I^{m}, \dot{I}^{m}\right) \rightarrow\left(S^{m}, s\right)$ which is univalent on $I^{m}-\dot{I}^{m}$, we define

$$
\begin{aligned}
\Phi:\left(I^{p}, \dot{I}^{p}\right) & \rightarrow\left(Y^{S^{m}}, v\right) \text { and } \Psi:\left(I^{n}, \dot{I}^{n}\right) \rightarrow\left(Y^{S^{m}}, v\right), \\
\Phi(\xi)(x) & =\phi(\xi) \quad \text { for }(\xi, x) \in I^{p} \times S^{m}, \\
\Psi(\eta)[\sigma(\zeta)] & =\psi(\eta, \zeta) \text { for }(\eta, \zeta) \in I^{n} \times I^{m} .
\end{aligned}
$$

If $[\langle\Phi\rangle,\langle\Psi\rangle]=0$ there exist continuous maps $\Omega_{t}: \dot{I}^{p+n} \rightarrow Y^{s^{m}}$ for $t \in I$ which form a homotopy such that

$$
\begin{aligned}
& \Omega_{0}(\xi, \eta)=\Phi(\xi) \text { for }(\xi, \eta) \in I^{p} \times \dot{I}^{n} \\
& \Omega_{0}(\xi, \eta)=\Psi(\eta) \text { for }(\xi, \eta) \in \dot{I}^{p} \times I^{n}
\end{aligned}
$$


and $\Omega_{1}: \dot{I}^{p+n} \rightarrow\{v\}$. It follows that the continuous maps

$$
\begin{array}{ll}
u_{t}: \dot{I}^{p+n} \rightarrow Y & \text { for } \quad t \in I, \\
u_{t}(\xi, \eta)=\Omega_{t}(\xi, \eta)(s) & \text { for }(\xi, \eta) \in \dot{I}^{p+n}
\end{array}
$$

form a homotopy such that

$$
u_{0}(\xi, \eta)=\phi(\xi) \text { for }(\xi, \eta) \in \dot{I}^{p+n},
$$

and hence that the maps $u_{t}$ have continuous extensions

$$
U_{t}: I^{p+n} \rightarrow Y \text { for } t \in I
$$

which form a homotopy such that

$$
U_{0}(\xi, \eta)=\phi(\xi) \text { for }(\xi, \eta) \in I^{p+n}, \quad U_{1}:\left(I^{p+n}, \dot{I}^{p+n}\right) \rightarrow(Y, y) .
$$

Finally the continuous maps

$$
\begin{array}{rlrl}
\omega_{t}: \dot{I}^{p+n+m} & \rightarrow Y & \text { for } \quad t \in I, \\
\omega_{t}(\xi, \eta, \zeta) & =U_{t}(\xi, \eta) & & \text { for }(\xi, \eta, \zeta) \in I^{p+n} \times \dot{I}^{m}, \\
\omega_{t}(\xi, \eta, \zeta) & =\Omega_{t}(\xi, \eta)[\sigma(\zeta)] & \text { for }(\xi, \eta, \zeta) \in \dot{I}^{p+n} \times I^{m}
\end{array}
$$

form a homotopy such that

$$
\left\langle\omega_{0}\right\rangle=[\langle\phi\rangle,\langle\psi\rangle] \neq 0 \text { and }\left\langle\omega_{1}\right\rangle=\left[\left\langle U_{1}\right\rangle,\langle v \circ \sigma\rangle\right]=0 .
$$

Since this is impossible, $[\langle\Phi\rangle,\langle\Psi\rangle] \neq 0$.

Next we deduce the following corollary:

If $y \in Y, w: S^{q} \times S^{m} \rightarrow\{y\}, q \geqq 1, p \geqq 1, n \geqq 1, m \geqq 1$ and

$$
\left[\pi_{q+p}(Y, y), \pi_{n+m}(Y, y)\right] \neq 0,
$$

then

$$
\left[\pi_{p}\left(Y^{s^{q} \times S^{m}}, w\right), \pi_{n}\left(Y^{s^{q} \times S^{m}}, w\right)\right] \neq 0 .
$$

Two applications of our previous result yield successively

$$
\left[\pi_{q+p}\left(Y^{s^{m}}, v\right), \pi_{n}\left(Y^{s^{m}}, v\right)\right] \neq 0, \quad\left[\pi_{p}\left(\left(Y^{s^{m}}\right) s^{q}, v^{\prime}\right), \pi_{n}\left(\left(Y^{s^{m}}\right) S^{q}, v^{\prime}\right)\right] \neq 0,
$$

where $v^{\prime}: Y^{s^{m}} \rightarrow\{v\}$, and to obtain the corollary we simply observe that $v^{\prime}$ corresponds to $w$ under the canonical homeomorphism mapping $\left(Y^{s^{m}}\right) s^{q}$ onto $Y^{s^{q}} X^{s^{m}}$.

Now consider for instance the special case in which $Y=S^{2}$. Since

$$
\left[\pi_{2}\left(S^{2}\right), \pi_{2}\left(S^{2}\right)\right] \neq 0,
$$

our first proposition implies that $\left(\left(S^{2}\right)^{S 1}, v\right)$ is not 2-simple, while the corollary implies that $\left({ }^{7}\right)$

( ${ }^{7}$ The referee pointed out to us that the structure of this group has been completely determined by W. G. Barratt, on p. 95 of the paper cited in footnote 2 . 


$$
\pi_{1}\left(\left(S^{2}\right)^{S^{1} \times S^{1}}, w\right)
$$

is non-abelian. Here $v$ and $w$ are constant maps.

12. The groups $\pi_{p}\left(Y^{x}, v\right) \otimes R$. We readopt the assumptions of $\S \S 2-10$, with $\operatorname{dim} X=Q<\infty$, and let $R$ be the field of rational numbers. Using the normal chain of $\S 8$ we see that

$$
\pi_{p}\left(Y^{\mathbb{X}}, v\right) \otimes R \simeq \underset{q=0}{\oplus} C_{p, q}^{(\infty)} \otimes R \text { for } p \geqq 2 .
$$

In case $\pi_{1}\left(Y^{x}, v\right)$ is abelian this formula holds also for $p=1$. Since

$$
C^{(n)} \otimes R=H\left(C^{(n-1)}, d^{(n-1)}\right) \otimes R \simeq H\left(C^{(n-1)} \otimes R, d^{(n-1)} \otimes i\right)
$$

for $n \geqq 1$, where $i$ is the identity map of $R, C^{(\infty)} \otimes R$ is the terminal group of the spectral sequence with the $n$th term

$$
\left(C^{(n)} \otimes R, d^{(n)} \otimes i\right)
$$

for $n \geqq 0$. The first two groups occurring in this sequence have the components

$$
C_{p, q}^{(0)} \otimes R \simeq C^{q}\left[K, \pi_{p+q}(Y) \otimes R\right], \quad C_{p, q}^{(1)} \otimes R \simeq H^{q}\left[X, \pi_{p+q}(Y) \otimes R\right] .
$$

13. A special obstruction. Suppose $m$ is an even positive integer, $S^{m}$ is an $m$-sphere, $s \in S^{m}, \sigma:\left(I^{m}, \dot{I}^{m}\right) \rightarrow\left(S^{m}, s\right)$ is a continuous map which is univalent on $I^{m}-\dot{I}^{m}$, and

$$
\mu \in H^{m}\left[S^{m}, \pi_{m}\left(S^{m}, s\right)\right]
$$

is the cohomology class of a cocycle with the value $\langle\sigma\rangle$ at $\sigma$.

The Whitehead product pairs $\pi_{m}\left(S^{m}, s\right)$ with itself to $\pi_{2 m-1}\left(S^{m}, s\right)$. Using the corresponding cup product we shall prove:

If $u: K_{2 m-1} \rightarrow S^{m}$ is a continuous map with the obstruction cocycle

$$
\Gamma \in C^{2 m}\left[K, \pi_{2 m-1}\left(S^{m}\right)\right],
$$

if all the values of $\Gamma$ lie in the subgroup of $\pi_{2 m-1}\left(S^{m}\right)$ generated by $[\langle\sigma\rangle,\langle\sigma\rangle]$, and if $j^{*}(\nu)=u^{*}(\mu)$ where

$$
j^{*}: H^{m}\left[X, \pi_{m}\left(S^{m}\right)\right] \simeq H^{m}\left[K_{2 m-1}, \pi_{m}\left(S^{m}\right)\right]
$$

is induced by the inclusion $K_{2 m-1} \subset X$, then the cohomology class of $2 \Gamma$ in $H^{2 m}\left[X, \pi_{2 n_{-1}-1}\left(S^{m}\right)\right]$ equals $\nu \cup \nu$.

Following the method of [5] we first consider a special case. Attaching $I^{2 m}$ to $S^{m}$ along $\dot{I}^{2 m}$ by the standard map representing $[\langle\sigma\rangle,\langle\sigma\rangle]$ we form the space $\bar{X}$ of a cell complex $\bar{K}$ with three cells: the vertex $s$, the $m$-cell $\sigma$ and a $2 m$-cell $\tau$ such that

$$
\begin{aligned}
& \tau(\xi, \eta)=\sigma(\xi) \text { for }(\xi, \eta) \in I^{m} \times \dot{I}^{m} \\
& \tau(\xi, \eta)=\sigma(\eta) \text { for }(\xi, \eta) \in \dot{I}^{m} \times I^{m}
\end{aligned}
$$


Letting $\bar{\Gamma}$ be the obstruction cocycle associated with the identity map $\bar{u}: \bar{K}_{2 m-1}=S^{m} \rightarrow S^{m}$, one readily verifies the above formula for the cohomology class of $2 \bar{\Gamma}$, for instance by using the formula for cup products in cubical singular homology theory given in [3].

To obtain the general case we simply extend $u$ to a continuous map $U: K_{2 m} \rightarrow \bar{X}$ and make use of the fact that $U^{*}$ maps the cohomology class of $\bar{\Gamma}$ onto the cohomology class of $\Gamma$.

14. The groups $\pi_{p}\left(S^{m^{X}}, v\right) \otimes R$. Suppose $Y=S^{m}$, an $m$-sphere, where $m \geqq 1$. In view of the complete determination of $\pi_{n}\left(S^{m}\right) \otimes R$ made in [3], the formulae of $\$ 12$ lead to the following results:

If $m$ is odd, then

$$
C_{p, q}^{(1)} \otimes R=\{0\} \text { unless } q=m-p, C_{p, m-p}^{(1)} \otimes R \simeq H^{m-p}(X, R),
$$

and $d^{(n)} \otimes i$ is trivial for $n \geqq 1$, hence

$$
\pi_{p}\left(Y^{X}, v\right) \otimes R \simeq H^{m-p}(X, R) \text { for } p \geqq 2 .
$$

If $m$ is even, then

$$
\begin{gathered}
C_{p, q}^{(1)} \otimes R=\{0\} \text { unless } q=m-p \text { or } q=2 m-1-p, \\
C_{p, m-p}^{(1)} \otimes R \simeq H^{m-p}(X, R), \quad C_{p, 2 m-1-p}^{(1)} \otimes R \simeq H^{2 m-1-p}(X, R),
\end{gathered}
$$

and the only nontrivial higher differential operator is

$$
d^{(m-1)} \otimes i: C_{p, m-p}^{(m-1)} \otimes R \rightarrow C_{p-1,2 m-p}^{(m-1)} \otimes R .
$$

We shall determine the corresponding homomorphism of $H^{m-p}(X, R)$ into $H^{2 m-p}(X, R)$.

We fix $s, \sigma, \mu$ as in the preceding section, choose a positive integer $k$ such that multiplication by $k$ maps $\pi_{2 m-1}\left(S^{m}, s\right)$ into the subgroup generated by $[\langle\sigma\rangle,\langle\sigma\rangle]$, and suppose that $v\left(K_{m-1}\right)=\{s\}$, as we may in view of $\S 9$. Then we consider the commutative diagram:

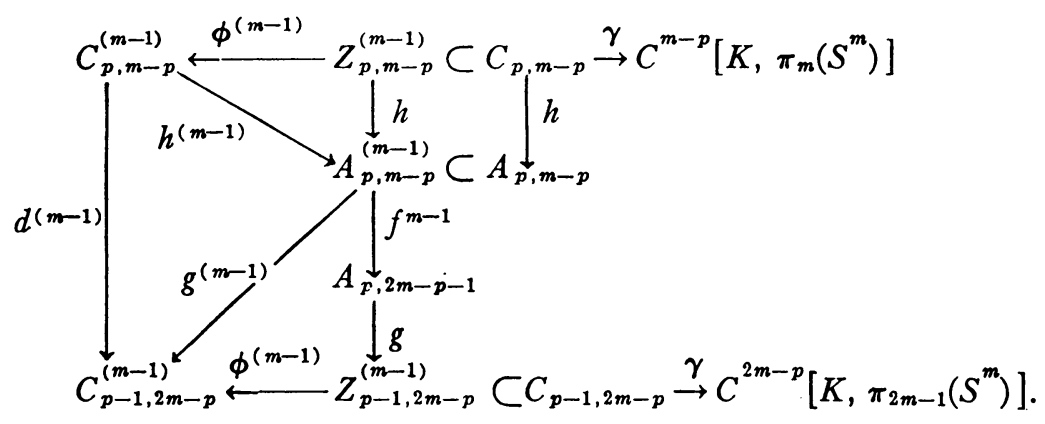
whence

Assuming that $c \in Z_{p, m-n}^{(m-1)}$ we choose $a \in A_{p, 2 m-p-1}$ so that $f^{m-1}(a)=h(c)$, 


$$
d^{(m-1)}\left[\phi^{(m-1)}(c)\right]=\phi^{(m-1)}[g(a)] .
$$

We also let

$$
\alpha \in H^{m-p}\left[X, \pi_{m}\left(S^{m}\right)\right] \text { and } \beta \in H^{2 m-p}\left[X, \pi_{2 m-1}\left(S^{m}\right)\right]
$$

be the cohomology classes of $\gamma(c)$ and $\gamma[g(a)]$. Since $f^{m-1}(k a)=h(k c)$ we may furthermore choose a continous map $w: I^{p} \times K_{2 m-p-1} \rightarrow S^{m}$ such that

$$
w(\xi, x)=v(x) \text { for }(\xi, x) \in \dot{I}^{p} \times K_{2 m-p-1} \cup I^{p} \times K_{m-p-1}
$$

and such that $w$ represents $k a$ while $w \mid I^{p} \times K_{m-p}$ represents $k c$. Then we consider on a $p$-sphere $S^{p}$ a cell complex with a single vertex $t \in S^{p}$ and a single $p$-cell $\tau:\left(I^{p}, \dot{I}^{p}\right) \rightarrow\left(S^{p}, t\right)$, combine this complex with $K$ to obtain a product complex $L$ on the space $S^{p} \times K_{2 m-p}$, and define the continuous map

$$
\begin{aligned}
u: L_{2 m-1} & =S^{p} \times K_{2 m-p-1} \cup\{t\} \times K_{2 m-p} \rightarrow S^{m}, \\
u[\tau(\xi), x] & =w(\xi, x) \text { for }(\xi, x) \in I^{p} \times K_{2 m-p-1}, \\
u(t, x) & =v(x) \text { for } x \in K_{2 m-p} .
\end{aligned}
$$

Using the description of $\gamma \circ g$ derived in $\S 6$ one verifies easily that the obstruction cocycle

$$
\Gamma \in C^{2 m}\left[L, \pi_{2 m-1}\left(S^{m}\right)\right]
$$

associated with $u$ is given by the formula

$$
\Gamma=(-1)^{p+1} \lambda \times(\gamma \circ g)(k a)=k(-1)^{p+1} \lambda \times(\gamma \circ g)(a)
$$

where $\lambda \in H^{p}\left(S^{p}\right)$ is the cohomology class of a cocycle with value 1 at $\tau$. Accordingly all the values of $\Gamma$ lie in the subgroup of $\pi_{2 m-1}\left(S^{m}\right)$ generated by $[\langle\sigma\rangle,\langle\sigma\rangle]$, and $\Gamma$ belongs to the cohomology class

$$
k(-1)^{p+1} \lambda \times \beta \in H^{2 m}\left[S^{p} \times K_{2 m-p}, \pi_{2 m-1}\left(S^{m}\right)\right] .
$$

It may also be checked quite directly that the cohomology class

$$
u^{*}(\mu) \in H^{m}\left[L_{2 m-1}, \pi_{m}\left(S^{(m)}\right)\right]
$$

is the image of the cohomology class

$$
\lambda \times k \alpha+e \times v^{*}(\mu) \in H^{m}\left[S^{p} \times K_{2 m-p}, \pi_{m}\left(S^{m}\right)\right]
$$

where $e \in H^{0}\left(S^{p}\right)$ is the unit cohomology class. From the result of $\S 13$ it follows that

$$
\begin{aligned}
2 k(-1)^{p+1} \lambda & \times \beta=\left[\lambda \times k \alpha+e \times v^{*}(\mu)\right] \cup\left[\lambda \times k \alpha+e \times v^{*}(\mu)\right] \\
& =(-1)^{p} k^{2}(\lambda \cup \lambda) \times(\alpha \cup \alpha)+e \times v^{*}(\mu \cup \mu)+2 k \lambda \times\left[\alpha \cup v^{*}(\mu)\right] \\
& =2 k \lambda \times\left[\alpha \cup v^{*}(\mu)\right]
\end{aligned}
$$

because $\lambda \cup \lambda=0$ and $\mu \cup_{\mu}=0$. Cancelling $\lambda$ we obtain the formula 


$$
2 k \beta=2 k \alpha \cup(-1)^{p+1} v^{*}(\mu),
$$

which shows that the differential operator $d^{(m-1)}$ can be interpreted cohomologically, up to elements of order $2 k$, as cup product multiplication by $(-1)^{p+1} v^{*}(\mu)$.

Observing that the homomorphisms

$$
\pi_{m}\left(S^{m}\right) \rightarrow R, \quad \pi_{2 m-1}\left(S^{m}\right) \rightarrow R
$$

which map $\langle\sigma\rangle$ and $[\langle\sigma\rangle,\langle\sigma\rangle]$ into 1 transform the Whitehead product into ordinary multiplication in $R$, and hence transform the cup product used above into the ordinary cup product with rational coefficients, we obtain the commutative diagram

$$
\begin{aligned}
& C_{p, m-p}^{(m-1)} \otimes R \simeq H^{m-p}(X, R) \\
& d^{(m-1)} \otimes i \downarrow \quad \downarrow U(-1)^{p+1} v^{*}\left(\mu^{\prime}\right) \\
& C_{p-1,2 m-p}^{(m-1)} \otimes R \simeq H^{2 m-p}(X, R)
\end{aligned}
$$

where $\mu^{\prime} \in H^{m}\left(S^{m}, R\right)$ is the cohomology class of a cocycle with value 1 at $\sigma$.

Therefore if $p \geqq 2$ the exact sequence

$$
\{0\} \rightarrow C_{p, 2 m-p-1}^{(\infty)} \otimes R \rightarrow \pi_{p}\left(S^{m^{X}}, v\right) \otimes R \rightarrow C_{p, m-p}^{(\infty)} \otimes R \rightarrow\{0\}
$$

leads us finally to the exact sequence

$$
\begin{array}{r}
H^{m-p-1}(X, R) \stackrel{\mathrm{U}^{*}\left(\mu^{\prime}\right)}{\longrightarrow} H^{2 m-p-1}(X, R) \rightarrow \pi_{p}\left(S^{m X}, v\right) \otimes R \\
\rightarrow H^{m-p}(X, R) \stackrel{\bigcup_{v^{*}\left(\mu^{\prime}\right)}^{\longrightarrow} H^{2 m-p}(X, R) .}{ }
\end{array}
$$

\section{REFERENCES}

1. W. S. Massey, Exact couples in algebraic topology, Ann. of Math. vol. 56 (1952) pp. 363396 and vol. 57 (1953) pp. 248-286.

2. - Products in exact couples, Ann. of Math. vol. 59 (1954) pp. 558-569.

3. J.-P. Serre, Homologie singulière des espaces fibrés, Ann. of Math. vol. 54 (1951) pp. 425505.

4. - Groupes d'homotopie et classes de groupes abéliens, Ann. of Math. vol. 58 (1953) pp. 258-294.

5. N. E. Steenrod, Products of cocycles and extensions of mappings, Ann. of Math. vol. 48 (1947) pp. 290-320.

6. The topology of fibre bundles, Princeton University Press, 1951.

7. G. W. Whitehead, On products in homotopy theory, Ann. of Math. vol. 47 (1946) pp. 460475.

8. - Homotopy theory, Massachusetts Institute of Technology, 1954.

9. J. H. C. Whitehead, Combinatorial homotopy (I), Bull. Amer. Math. Soc. vol. 55 (1949) pp. 213-245.

BROWN UNIVERSITY, Providence, R. I. 